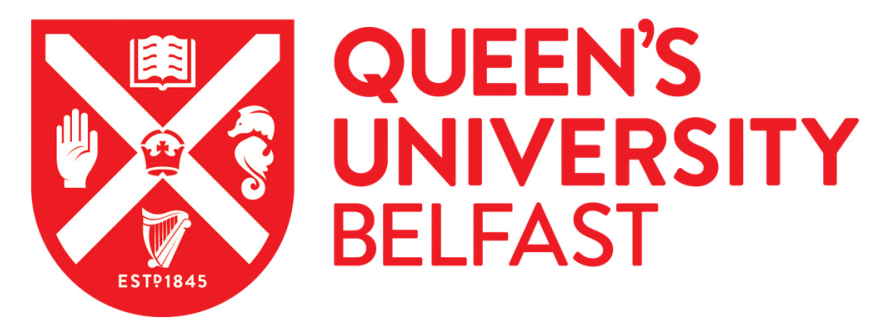

\title{
Detection and plasma assisted degradation of dye on reusable gold coated tungsten nanofuzz array surface-enhanced Raman scattering substrate
}

Liu, D., Liu, L., Ji, L., Qi, Z., Xia, Y., Song, Y., Dong, D., Li, Z., Liu, R., Liu, B., Sun, D., \& Liu, D. (2019).

Detection and plasma assisted degradation of dye on reusable gold coated tungsten nanofuzz array surfaceenhanced Raman scattering substrate. Applied Surface Science, 469, 262-268.

https://doi.org/10.1016/j.apsusc.2018.11.042

Published in:

Applied Surface Science

Document Version:

Peer reviewed version

Queen's University Belfast - Research Portal:

Link to publication record in Queen's University Belfast Research Portal

Publisher rights

(C) 2018 Elsevier Ltd.

This manuscript version is made available under the CC-BY-NC-ND 4.0 license http://creativecommons.org/licenses/by-nc-nd/4.0/,which permits distribution and reproduction for noncommercial purposes, provided the author and source are cited

\section{General rights}

Copyright for the publications made accessible via the Queen's University Belfast Research Portal is retained by the author(s) and / or other copyright owners and it is a condition of accessing these publications that users recognise and abide by the legal requirements associated with these rights.

\section{Take down policy}

The Research Portal is Queen's institutional repository that provides access to Queen's research output. Every effort has been made to ensure that content in the Research Portal does not infringe any person's rights, or applicable UK laws. If you discover content in the

Research Portal that you believe breaches copyright or violates any law, please contact openaccess@qub.ac.uk. 


\title{
Detection and plasma assisted degradation of dye on reusable gold coated tungsten nanofuzz array surface-enhanced Raman scattering substrate
}

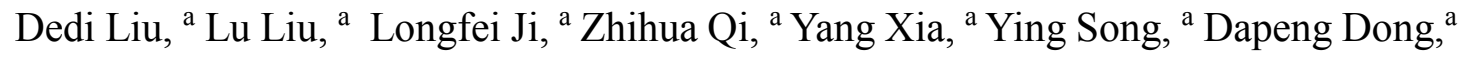
Zhenghua Li, ${ }^{\text {a }}$ Ran Liu, ${ }^{\mathrm{b}}$ Bingbing Liu, ${ }^{\mathrm{b}}$ Dan Sun, ${ }^{\mathrm{c}}$ Dongping Liu ${ }^{\mathrm{a}, *}$

${ }^{a}$ School of Physics and Materials Engineering, Dalian Minzu University, Dalian 116600, People's Republic of China.

${ }^{\mathrm{b}}$ State Key Laboratory of Superhard Materials, Jilin University,Changchun 130012 , China.

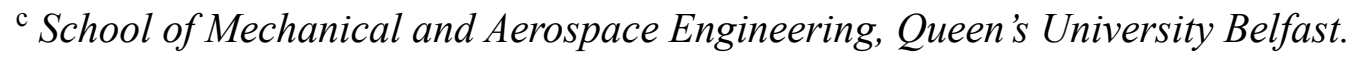

\begin{abstract}
A novel gold coated tungsten nanofuzz array (TNFA) substrate has been prepared by irradiating polycrystalline tungsten using radio frequency plasma, followed by gold sputter coating. The surface morphology of the tungsten can be modified by tuning the irradiation parameters. The surface-enhanced Raman scattering (SERS) effect of the as prepared substrates has been tested using different molecule probes, namely, Rhodamine B (RHB), brilliant blue (X-BR) and reactive brilliant red (X-3B). Results show that the TNFA substrate exhibit the highest dye detection sensitivity (as low as $<$ $10 \mathrm{ppb}$ ) and excellent repeatability. A simple room temperature atmospheric pressure plasma treatment can be used to clean the substrate for re-use purpose through complete oxidization/degradation of the dye species. Systematic study reveals that the SERS enhancement effect is strongly dependent on the substrate surface morphologies and the laser excitation wavelength used. In addition, the SERS activity achieved by our novel substrate can be applied to a variety of dye molecules, demonstrating its potential
\end{abstract}


to be generalized for a wider range of applications.

\section{Keywords}

SERS; nanofuzz array; plasma; dye; degradation

\section{Introduction}

For centuries, dyes have been used by human being to beautify the world. However, since the discovery of the first synthetic dye in the late $19^{\text {th }}$ century [1], the large-scale production and extensive applications of synthetic dyes have brought about considerable impacts to the earth ecology, leading to serious concerns in environmental pollution and water safety [2-4]. There is an urgent need to effectively eliminate dyes from their production waste $[5,6]$, however, the detection and identification on traces of dye components is extremely challenging. The weak spectral and chemical signals can be hardly detected by any conventional chromatographic and spectroscopic tools that require sizable amount of samples $[7,8]$. Some non-destructive characterization techniques such as Fourier-Transform Infrared (FTIR) and Raman/FTIR also suffer from weak signals or low sensitivity towards sub-nanogram samples [9].

The discovery of surface-enhanced Raman scattering (SERS) effect in 1974 [10] and the subsequent development of such technology has allowed researchers to successfully detect and identify traces of dyes with high sensitivity and molecular specificity at the lowest reported detection concentration limits in the range of $10^{-}$ ${ }^{10} \mathrm{M} \sim 10^{-12} \mathrm{M}$ [11,12]. Single-molecule fingerprint-vibration information has been obtained for ultra-low concentration analyte (chemical and biological) molecules [6-8, 13-16]. As such, the use of SERS has attracted increasing attention from the scientific community and the advancement of this technology is expected to deliver significant impact to fundamental science as well as analytical applications. Electromagnetic mechanism (EM) and chemical mechanism (CM) were considered as the two main mechanisms of SERS $[17,18]$. It is widely accepted that the EM enhancement is the 
main origin and the primary contributing factor to the SERS effect [19]. The enhancement results from the amplification of the light by the excitation of localized surface plasmon resonances and the light concentration occurs preferentially in the gaps, crevices, or sharp features of plasmonic materials (e.g., $\mathrm{Au}, \mathrm{Ag}$, and $\mathrm{Cu}$ ) with nanoscale features [20].

The traditional metal nanoparticle aggregates within colloidal solutions have been reported to offer high SERS sensitivity, however, such system has limited stability and is not suitable for practical applications. For an efficient SERS-active chip, robust metallic nanostructures on solid substrates with open morphology is a more feasible option, as they can offer large specific surface area for analytes adsorption and provide a large density of hot spots within the laser-illumination area [20]. For instance, Shanmukh,et al., fabricated Ag nanorod SERS array substrate using the oblique angle deposition (OAD) method, and their substrate demostrated enhancement factors of greater than $10^{8}$. [13]. Vertically stacked 3D cross-point plasmonic nanostructures by nanotranfer printing technique have also been proposed. Depending on structural and material parameters, excellent enhancement of Raman signals from probed molecules with an average enhancement factor of $\approx 4.1 \times 10^{7}$ can be achieved [14]. Zheng et al., fabricated $\mathrm{Au}$ nanotriangle or nanohole based arrays fabricated by nanosphere lithography [21]. Ultrathin silver-coated gold nanoparticles were attempted as a substrate for SERS by Luca et al, which show high sensitivities on various probe molecules [22]. Some non-metallic materials have also been used as template for producing nanoarray structures. Vijayarangamuthu et al.[23] and Fan et al. [24] have investigated the SERS effect of porous silicon and Si nanorod array coated with gold film, respectively. Ag coated monolayer array of $\mathrm{SiO}_{2}$ spheres were also used as SERS substrate for organic dye detection.[25] These substrates showed high sensitivity and uniformity. Despite the rapid development in the fabrication of various nanoarray structures for SERS applications, the reported methods mostly involve complicated/time consuming and costly fabrication processes. In addition, maintaining detection stability and achieving reutilization of SERS substrates still remain challenges. 
In this work, we developed a simple and low cost method to fabricate a robust and re-usable SERS substrates with high sensitivity and reliability. A radio-frequency (RF) plasma was used to irradiate tungsten substrate to produce a novel tungsten nanofuzz array structure (TNFA), which was then coated with gold film through sputter coating. These gold coated-TNFAS were then used as SERS substrates for dye detection using various dye molecules. A gentle atmospheric pressure plasma treatment at room temperature can be used to effectively clean the post-detection substrate for re-use purpose.

Fig. 1 Schematic illustration of the fabrication and re-use of the GC-TNFAs substrate.

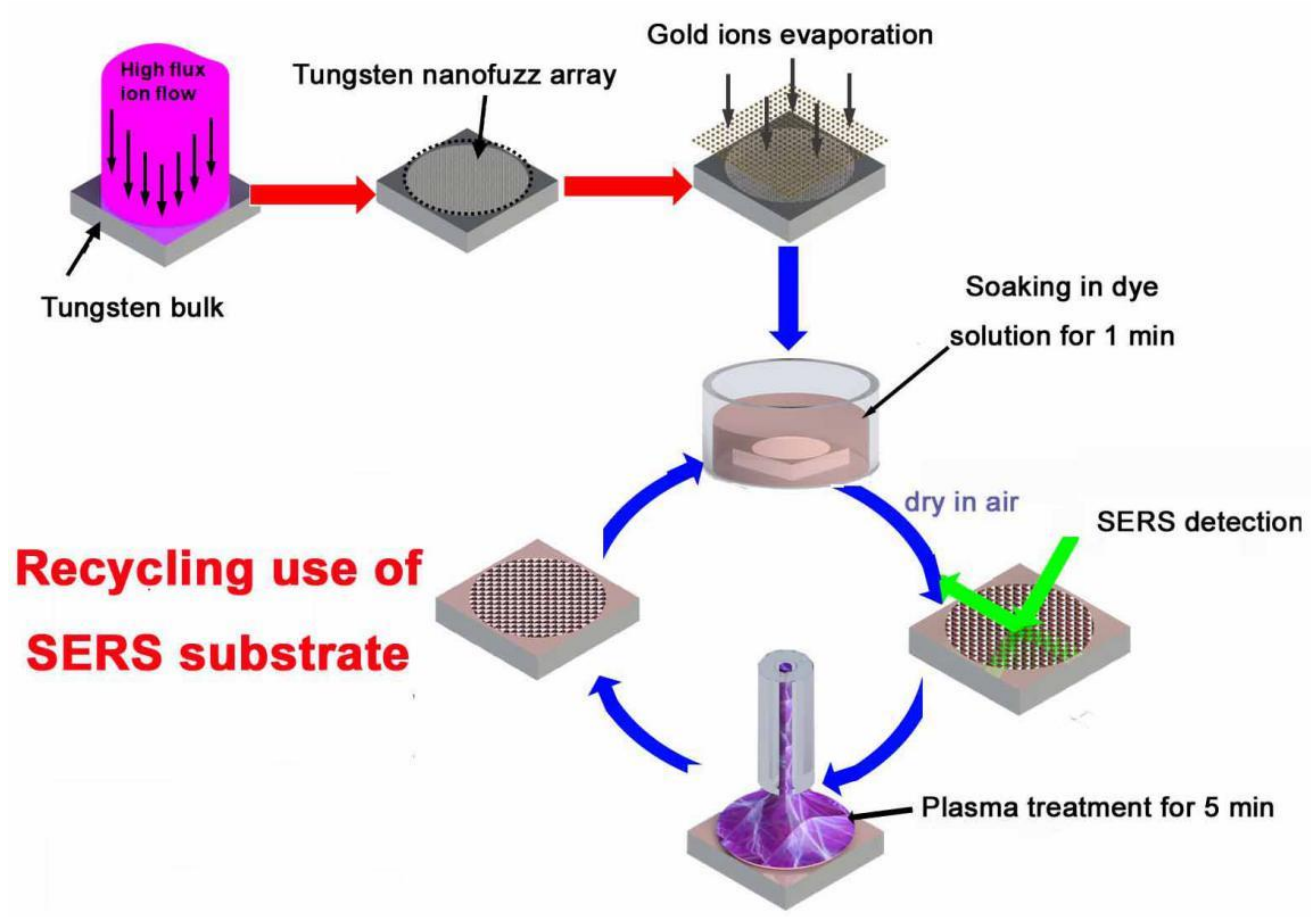

\section{Experimental methods}

\subsection{Fabrication of SERS substrate}

Polycrystalline tungsten specimens ( $99.99 \%$ purity) were purchased from Honglu Corporation, China. The as-purchased tungsten specimens were mechanically polished to a mirror-finish $(\mathrm{Rq}<0.1 \mu \mathrm{m})$. The polished specimens were then annealed in a vacuum oven $\left(1373 \mathrm{~K}, 10^{-5} \mathrm{~Pa}\right)$ for $2 \mathrm{hrs}$ to relieve the residual stress and reduce the surface defects. 
W was selected as an ideal rock-bottom SERS substrate, due to its high hardness, good thermal properties, high chemical stability, etc. However, these properties make it difficult to etch its surface to obtain nanostructure. And the bombardments of $\mathrm{W}$ by the high-flux and low-energy ions lead to the serious irradiation damages. In this work, an in-house RF plasma system operating with helium or hydrogen as operating gas has been deployed to irradiate the polished tungsten specimens. By selecting different gas and adjusting the selected gas ion fluxes, tungsten specimens with different nanostructured surface morphologies can be produced. The irradiation parameters deployed in this study can be found in Table 1. More details regarding the equipment used can be found from our previous publications [26,27].

\begin{tabular}{|c|c|c|c|c|c|}
\hline $\begin{array}{c}\text { Surface } \\
\text { Morphologies }\end{array}$ & $\begin{array}{c}\text { Plasma } \\
\text { Species }\end{array}$ & $\begin{array}{c}\text { Ion Energy } \\
(\mathrm{eV})\end{array}$ & $\begin{array}{c}\text { Ion } \\
\text { Fluence } \\
(\mathrm{ions} / \mathrm{m})\end{array}$ & $\begin{array}{c}\text { Ion Flux } \\
\left(\text { ions } / \mathrm{m}^{2} \cdot \mathrm{s}\right)\end{array}$ & $\begin{array}{c}\text { Surface } \\
\text { Temperature } \\
(\mathrm{K})\end{array}$ \\
\hline Nanofuzzes & Helium ions & 200 & $5.0 \times 10^{25}$ & $1.0 \times 10^{22}$ & 1590 \\
\hline Nanostripes & Helium ions & 200 & $5.0 \times 10^{25}$ & $5.0 \times 10^{21}$ & 1130 \\
\hline Nanoridges & $\begin{array}{c}\text { Hydrogen } \\
\text { ions }\end{array}$ & 200 & $5.0 \times 10^{25}$ & $6.0 \times 10^{21}$ & 1080 \\
\hline Nanowrinkles & $\begin{array}{c}\text { Hydrogen } \\
\text { ions }\end{array}$ & 200 & $5.0 \times 10^{25}$ & $2.0 \times 10^{21}$ & 1020 \\
& & & & \\
\hline
\end{tabular}

Table 1. Plasma irradiation conditions for creating different tungsten surface nanostructures

An ion sputter coater (Hitachi, e-1010) was used to deposit a thin layer of gold onto the plasma irradiated tungsten substrates for 90 seconds. The microstructures of specimens have been observed using a scanning electron microscope (SEM, Hitachi S4800) and the crystalline structures of the specimens were studied using X-ray diffraction (XRD, Rigaku D/max-RA), using CuKal radiation with $\lambda=0.15406 \mathrm{~nm}$. 


\subsection{SERS experiment}

A Renishaw inVia Reflex Raman spectrometer with $532 \mathrm{~nm}$ laser was used for the acquisition of Raman spectra from dye molecules adsorbed on different substrates. A LabRam HR Evolution system with different laser wavelengths (473 nm, $532 \mathrm{~nm}$ and $785 \mathrm{~nm}$ ) has been deployed to identify the optimized excitation wavelength for the SERS substrates. All the SERS spectra were collected at room temperature.

All samples, namely, gold coated as-polished tungsten (polished, no plasma irradiation, gold-coated), TNFAs (polished and plasma irradiated) and GC-TNFAs (polished, plasma irradiated and gold-coated) were first rinsed in deionized water and dried, then immersed in the RHB aqueous solution (concentration $=10 \mathrm{ppm}$ ) for $1 \mathrm{~min}$, and dried under ambient conditions prior to the spectrum acquisition. A $532 \mathrm{~nm}$ laser was used to acquire the Raman spectra from the traced dye adsorbed on the different substrates and elucidate the effect of different surface morphology on the SERS signals. Two other typical dyes commonly used in the industry, namely X-BR and X-3B, were also characterized following the same procedures.

The influence of dye concentration was investigated for the GC-TNFAs substrates using the $532 \mathrm{~nm}$ laser after the substrates have been immersed in different RHB aqueous solutions (10 ppm, $1 \mathrm{ppm}, 100 \mathrm{ppb}$ and $10 \mathrm{ppb}$, respectively, immersion time $=1 \mathrm{~min})$.

A typical sample (GC-TNFAs immersed in the $10 \mathrm{ppm}$ solution) was cleaned by exposing the sample in an atmospheric pressure helium (He) plasma for $5 \mathrm{~min}$, and the SERS characterization was repeated to verify the dye sensing repeatability of the substrate.

Finally, to further understand the wavelength dependence of the SERS effect, lasers with wavelengths of $473 \mathrm{~nm}, 532 \mathrm{~nm}$ and $785 \mathrm{~nm}$ were employed to investigate the Raman spectra of RHB on the GC-TNFAs substrates. 


\section{Results and discussions}

Fig 2 shows the surface morphology and structure comparison between gold-coated aspolished tungsten surface and the GC-TNFAS. It can be seen that the gold-coated aspolished bulk tungsten, Fig. 2(a), has a relatively smooth surface, whereas the GCTNFAS, (Fig.2 (b)), exhibits fuzz-like morphology, with the fuzz orientation perpendicular to the bulk surface. The average distance between the neighboring fuzzes is about $100 \mathrm{~nm}$, and the GC-TNFAS have a uniform diameter of about $50 \mathrm{~nm}$. From the insert of Fig.2 (b), we can we can clearly see that thin films with unique thickness of $\sim 10 \mathrm{~nm}$ were coated on the nanofuzzes. Furthermore, a cross-sectional image of GC-TNFAS, Fig. 2 (c), clearly shows that the warped nanofuzzes have a length of about $1.5 \mu \mathrm{m}$

$\mathrm{XRD}$ analysis has been carried out to investigate the crystalline structure of GCTNFAS, see Fig. 2 (d). Seven characteristic peaks with indices (110), (200), (211), (220), (310), (222) and (321), typical of tungsten BCC lattice, have been observed for both pristine tungsten and the TNFAs. This indicates the plasma irradiation induced TNFAs formation has led to any alteration to the tungsten BCC lattice and the lattice cell has dimension $\mathrm{a}=0.314 \mathrm{~nm}[28]$.

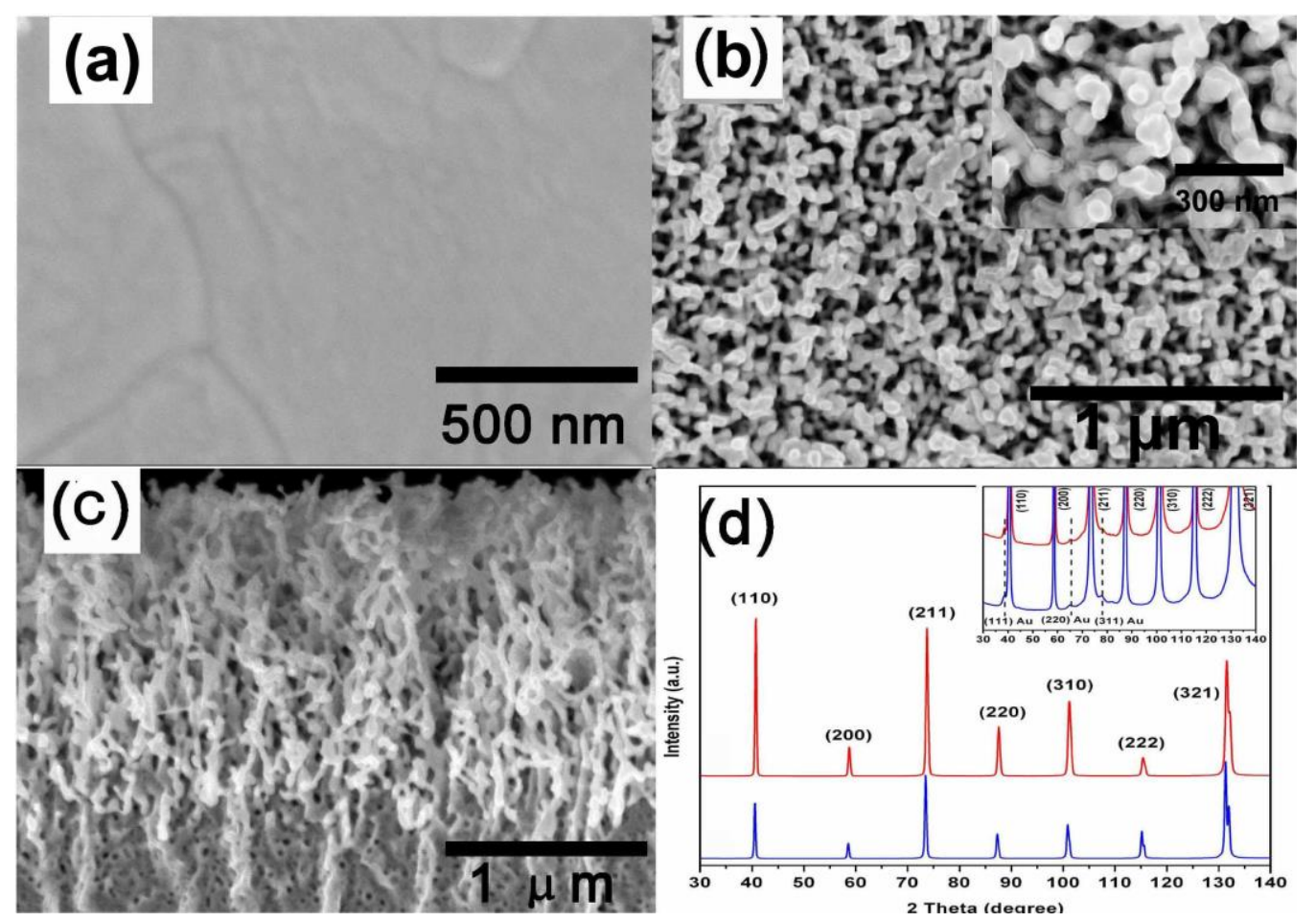


Fig. 2 SEM images of (a) gold-coated as-polished tungsten surface, (b) top view and (c) cross-sectional view of GC-TNFAS. (d) XRD patterns of gold coated as-polished tungsten surface (red) and TNFAs (blue).

A close comparison between the XRD patterns reveals the difference in the relative intensities of the diffraction peaks. For the as-polished tungsten (red), the strongest peak was from the (110) diffraction plane. However, in the case of TNFAs, the relative intensities of (110) and (220) peaks decreased significantly, and the strongest diffraction peak is from the (321) plane. This indicates that the atom packing density in (110) type planes has been reduced greatly. Our recent work on tungsten [26] suggested that tungsten atom layers between the (110) type planes strip during the plasma irradiation, due to the seeping of $\mathrm{He}$ atoms into the $\{101\}$ crystal planes. In this work, the decreasing of (110) diffraction peaks intensities suggests that the tungsten atoms on these planes were most susceptible to the plasma bombardment and removal, which is consistent with the previous findings [26].

To confirm the presence of gold films on the tungsten surfaces, a zoomed in new of the XRD patterns has been presented in Fig. 2 (d) inset. Three additional peaks with relatively weak intensity with indices (111), (220) and (311) have been observed for both samples. This is consistent with FCC gold with a lattice parameter $\mathrm{a}=0.235 \mathrm{~nm}$ [29], indicating that gold film has been successfully deposited on both tungsten substrates. To evaluate the Raman-enhancing capability of the GC-TNFAs, a RHB aqueous solution with a very low concentration (10 ppm) was used. RHB is chosen as it is very commonly found in organic dye pollutants and is known to give reliable SERS signals [30, 31]. As shown in Fig. 3(b), no obvious Raman signals can be detected for the TNFAs (no gold coating) substrate. In contrast, distinct peaks at $621 \mathrm{~cm}^{-1}, 934 \mathrm{~cm}^{-}$ ${ }^{1}, 1199 \mathrm{~cm}^{-1}, 1280 \mathrm{~cm}^{-1}, 1358 \mathrm{~cm}^{-1}, 1433 \mathrm{~cm}^{-1}, 1507 \mathrm{~cm}^{-1}, 1530 \mathrm{~cm}^{-1}, 1563 \mathrm{~cm}^{-1}$ and $1648 \mathrm{~cm}^{-1}$, have been observed for the GC-TNFAS substrate shown in Fig. 3(a). All of these peaks were characteristic of RHB [32,33], indicating GC-TNFAS substrate is highly sensitive towards probing the dye molecular signature. 


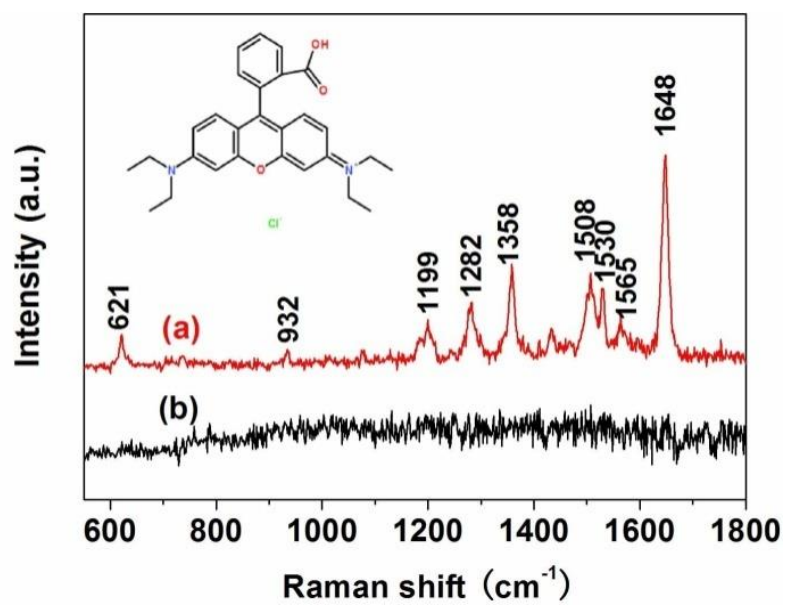

Fig. 3 Raman spectra of RHB on the (a) GC-TNFAs and (b) TNFA substrates

The influence of surface morphology on the SERS sensitivity of gold coated tungsten has been investigated using the $532 \mathrm{~nm}$ laser with the power of $0.2 \mathrm{~mW}$. Fig. 4 shows the Raman spectra of RHB on tungsten substrates with different surface morphologies, including gold coated TNFAs, nanostripe array, nanoridge array, nanowrinkle and smooth bulk substrates. Raman spectra show characteristic peaks of RHB on most substrates. However, the intensity of Raman peaks varies considerably, depending on the the substrate surface microstructures. The corresponding SEM images of each substrate can be found on the left of Fig. 4. The intensities of Raman peaks is the strongest for the GC-TNFAs substrate, followed by nanostripe array, nanoridge array, nanowrinkle and the as-polished substrate. The RHB Raman intensity of the GCTNFAs substrate is about 100 times greater than that of the as-polished substrate. This maybe be partly due to the nanofuzz structure has the greatest surface roughness/specific surface area, which facilitate greater analyte absorption and hence notably enhances the Raman signals. However, we could not test the surface roughness of the nanofuzz array substrate, due to the small diameters of the nanofuzzes which were equal to the tips of the AFM probe. But obviously, such nanostructured substrate has the highest surface roughness, according to the cross-sectional view SEM image shown in Fig. 2 c. Therefore, it is reasonable to conclude that the SERS activities depend on the substrate surface roughness. 


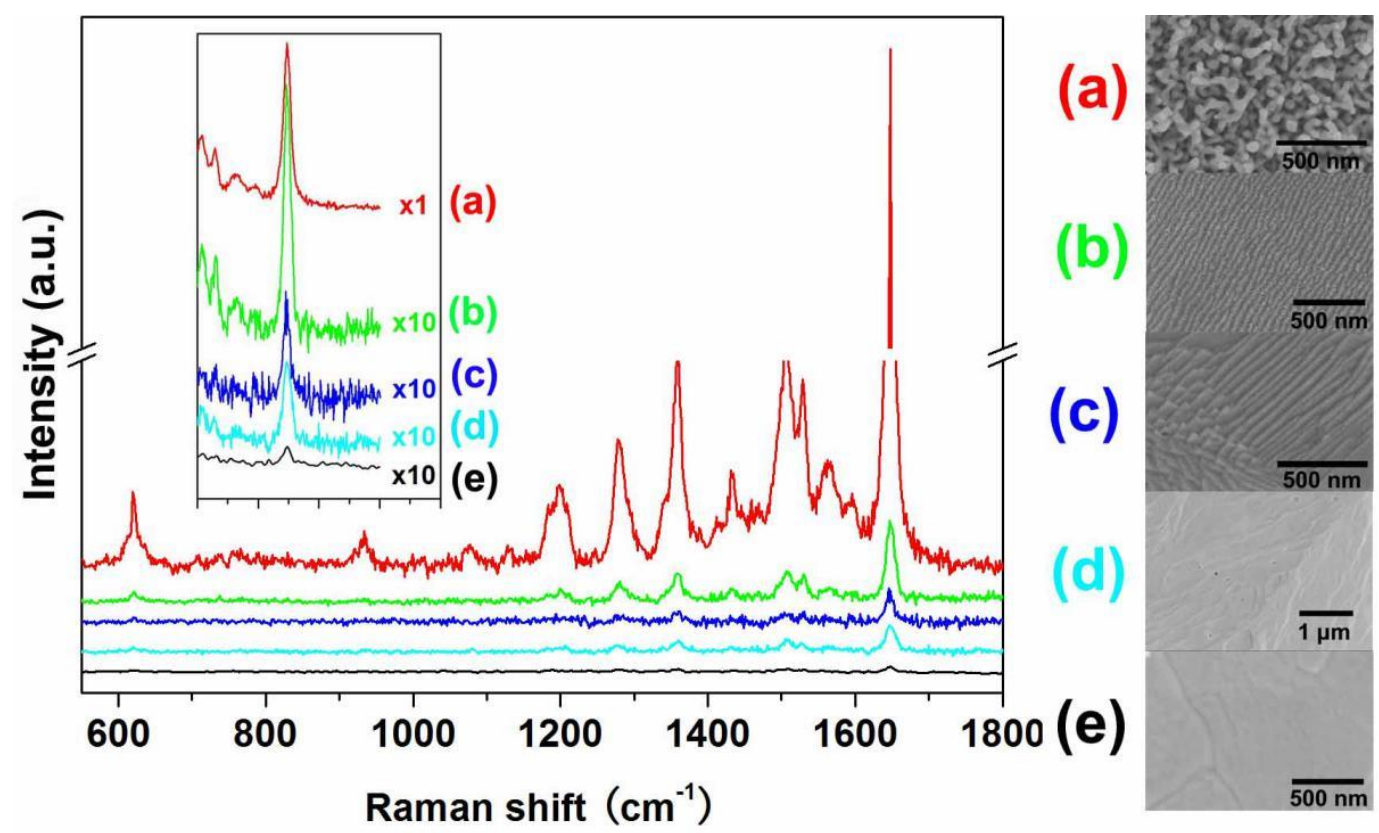

Fig. 4 Raman spectra of RHB on gold-coated tungsten with different surface morphologies: (a) nanofuzz array, (b) nanostripe array, (c) nanoridge array, (d) nanowrinkle and (e) as-polished tungsten substrate. The Raman intensities of b, c, d and e shown in the insert have been all multiplied by 10 .

Fig 4 suggests that GC-TNFAs substrate is the best performing substrate comparing to other substrates with different surface morphology. Therefore, it is further tested using different concentrations of RHB (ranging from $10 \mathrm{ppm}$ to $10 \mathrm{ppb}$ )) to explore its detection limit. As can be seen from Fig. 5, the Raman intensities gradually decrease with decreasing RHB concentrations and the lowest detection limit for RHB is $\sim 10 \mathrm{ppb}$.

To investigate whether the GC-TNFAS substrate could be reused, the substrate (rinsed in deionized water and dried, followed by 10 min immersion in $10 \mathrm{ppm} \mathrm{RHB}$ ) was subjected to an atmospheric pressure plasma (He with gas flow rate of 1 SLM and at an alternating voltage of $20 \mathrm{kV}$ ) at room temperature for 5 minutes. The Raman spectra for GC-TNFAS substrate before (black) and after (red) the plasma cleaning treatment are shown in Fig. 6 (a). It can be clearly seen that all characteristic peaks signifying RHB diminished completely after the plasma treatment, indicating that the dye molecules absorbed on the GC-TNFAS surface have been decomposed/removed completely during atmospheric pressure plasma treatment. The plasma treated GC- 
TNFAS substrate was re-tested during the subsequent plasma cleaning and reimmersion cycles, and strong and consistent SERS signals can be obtained even after multiple cycles, indicating the competency of GC-TNFAs for re-utilization. Furthermore, the SEM image of the plasma treated GC-TNFAS substrate (Fig 6 (b)) shows that the morphology of the substrate remains unchanged after multiple plasma cleaning cycles, indicating the plasma treatment used was a gentle process which does not alter/ destroy the substrate structure.

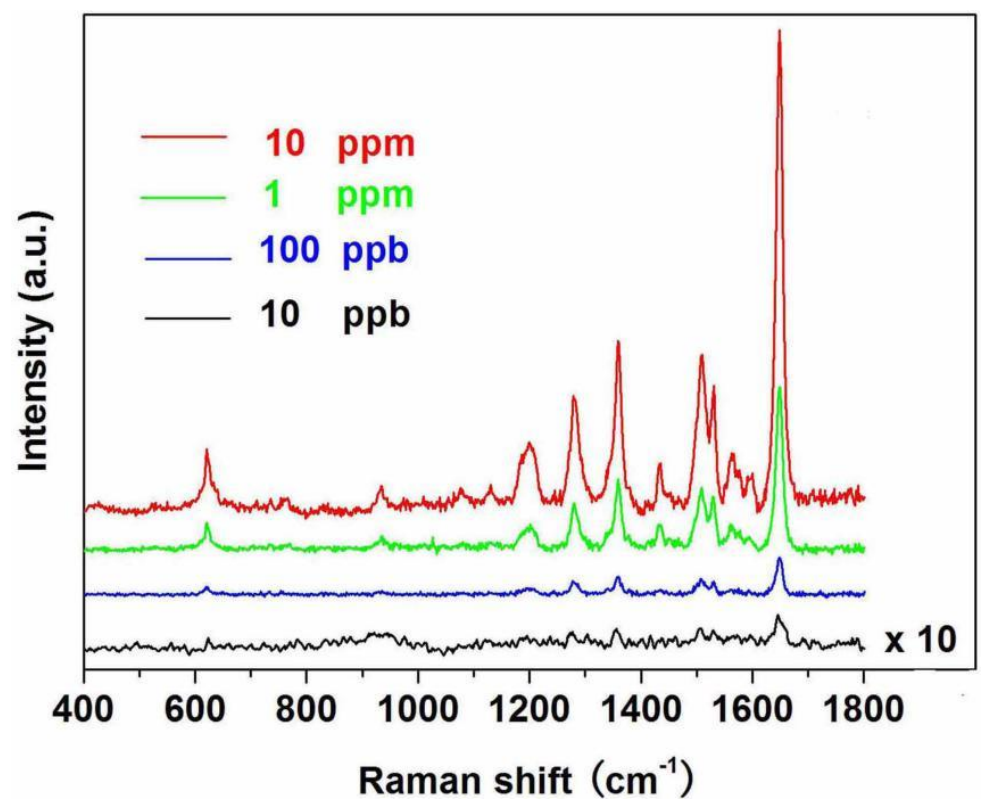

Fig. 5 Raman spectra of GC-TNFAS immersed in RHB with different concentrations. The intensity for the $10 \mathrm{ppb}$ sample has been multiplied by 10 .

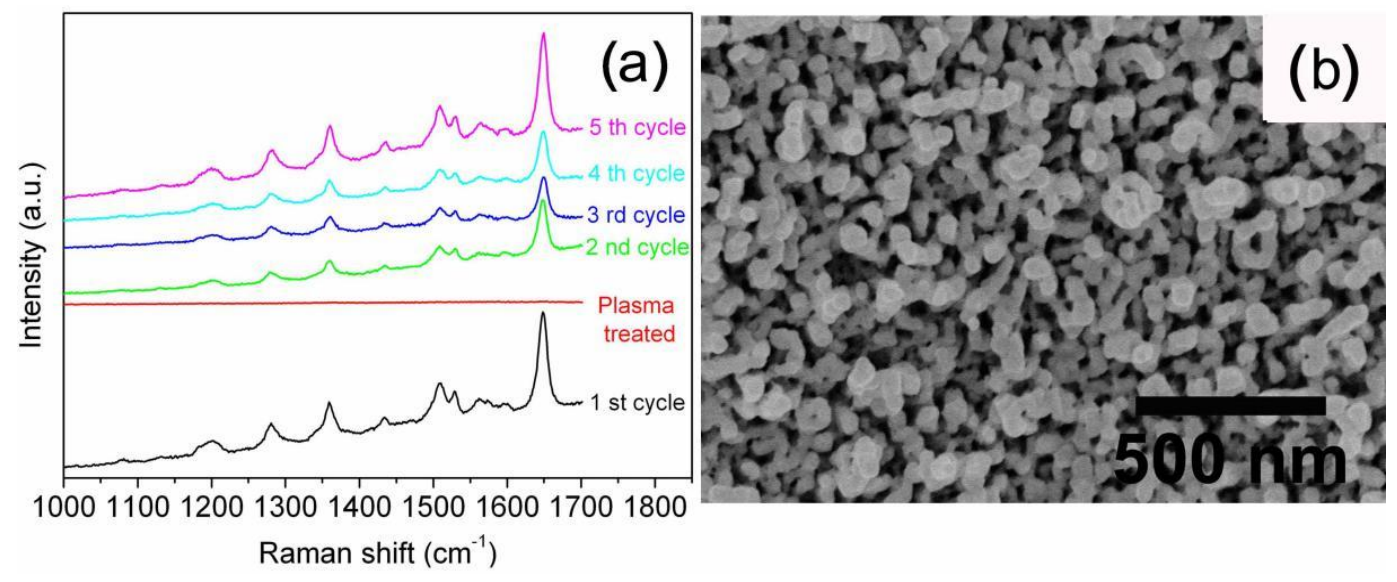

Fig. 6 (a) RHB Raman spectra collected during five plasma cleaning/re-immersion cycles. (b) SEM image of GC-TNFAS substrate after the plasma cleaning treatment. 
A typical emission spectrum collected during the atmospheric pressure plasma cleaning process has been collected, see Fig.7. The major emission lines are from $\mathrm{N}_{2}$ $(\mathrm{C} \rightarrow \mathrm{B}), \mathrm{N}_{2}{ }^{+}(\mathrm{B} \rightarrow \mathrm{X}), \mathrm{He}^{*}$, and $\mathrm{O}^{*}$ [34,35]. In particular, several sharp lines corresponding to UV radiation $(318,338,358,382$ and $392 \mathrm{~nm})$ have been observed. It is known that $\mathrm{O}$ radicals play an active role in the oxidation of organic molecules $[35,36]$. UV radiation can also lead to dye degradation [37]. The cleaning power of the atmospheric pressure plasma can be attributed to the combined effect of the plasmagenerated $\mathrm{O}$ radicals as well as the strong $\mathrm{UV}$ radiation. On the other hand, the substrate materials (gold and tungsten) both remain stable when subjected to O radicals and UV radiation.

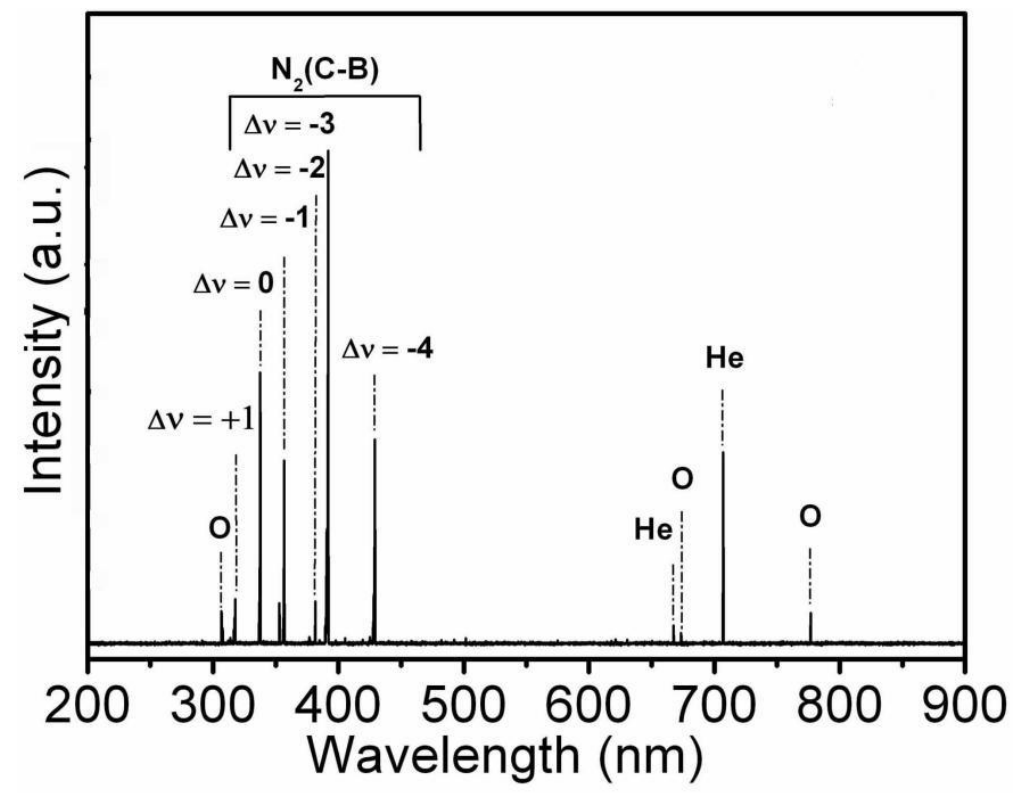

Fig 7. Typical emission spectrum of the atmospheric pressure plasma used for substrate cleaning.

The capability of different tungsten substrates in detecting other analysts has also been explored. Fig. 8 shows the Raman spectra of X-BR and X-3B (10 ppm) adsorbed on the GC-TNFAs (red), gold-coated as-polished tungsten (blue), and (c) as polished pristine tungsten (black) substrates under $532 \mathrm{~nm}$ laser excitation wavelength. The molecular structure of each dye has been given in the figure inset. Compared to the aspolished pristine and gold-coated pristine tungsten substrates, the GC-TNFAs substrate demonstrated greatly enhanced SERS signal for both types of dye molecules tested. 
This is evidenced by the intensive characteristic peaks shown for X-BR $(177,301,725$, 1098, 1339, 1448, 1526, 1545 and $\left.1618 \mathrm{~cm}^{-1}\right)$ and for X-3B $(145,396,511,593,638$, $681,747,839,951,1038,1137,1340,1451,1526$ and $\left.1593 \mathrm{~cm}^{-1}\right)$.
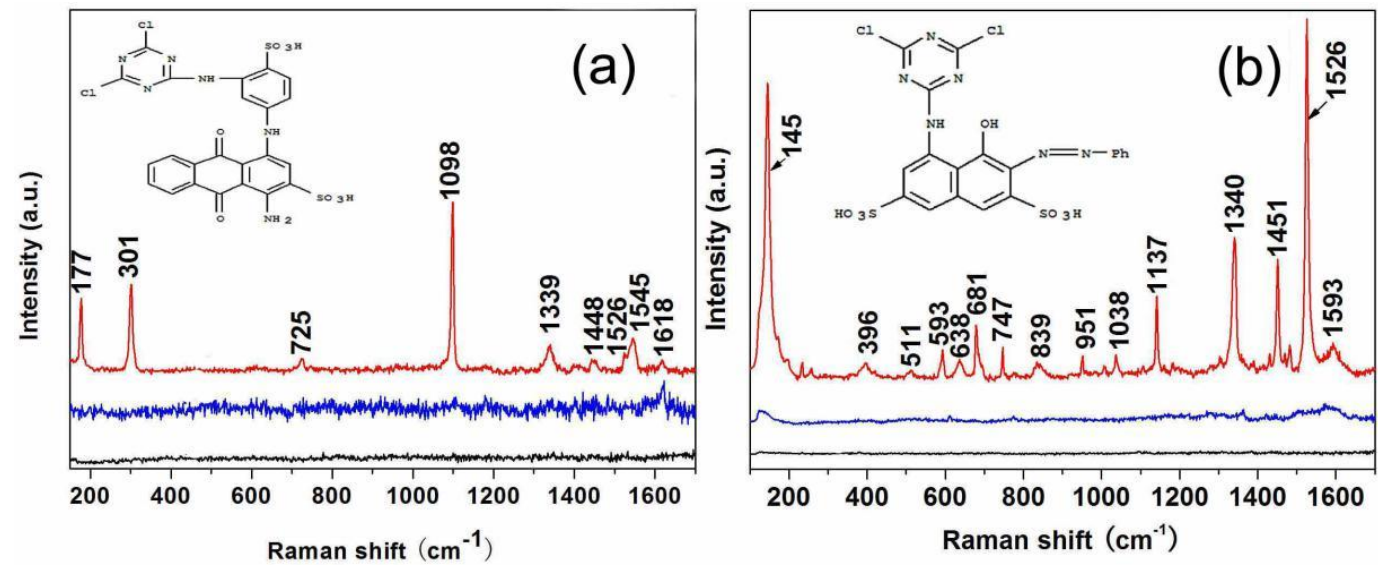

Fig. 8 Raman spectra of different tungsten substrates immersed in 10 ppm (a) X-BR and (b) X-3B. Red line: GC-TNFAs blue line: gold-coated as-polished bulk tungsten surface and black line: as-polished pristine tungsten substrates.

To further explore the role of laser wavelength on the SERS effect and develop more in-depth understanding of the mechanisms involved, Raman spectra have been collected for different laser excitation wavelengths (473 nm, $532 \mathrm{~nm}$ and $785 \mathrm{~nm}$ ) with the standard Raman probe RHB (shown in Fig 9 (a)(b)(c)). In line with the previous discussions, for wavelengths $532 \mathrm{~nm}$, see Fig. 9 (b), the GC-TNFAs substrate demonstrated a much greater sensitivity compared to the other two as-polished bulk substrates (with or without gold coating). However, when the $785 \mathrm{~nm}$ laser was applied, the SERS effect of GC-TNFAS was less distinct. For the $473 \mathrm{~nm}$ laser, the SERS effect of GC-TNFAs substrate is similar to that of gold-coated as-polished tungsten substrate, showing only slightly enhanced signals compared to the as-polished pristine tungsten. 

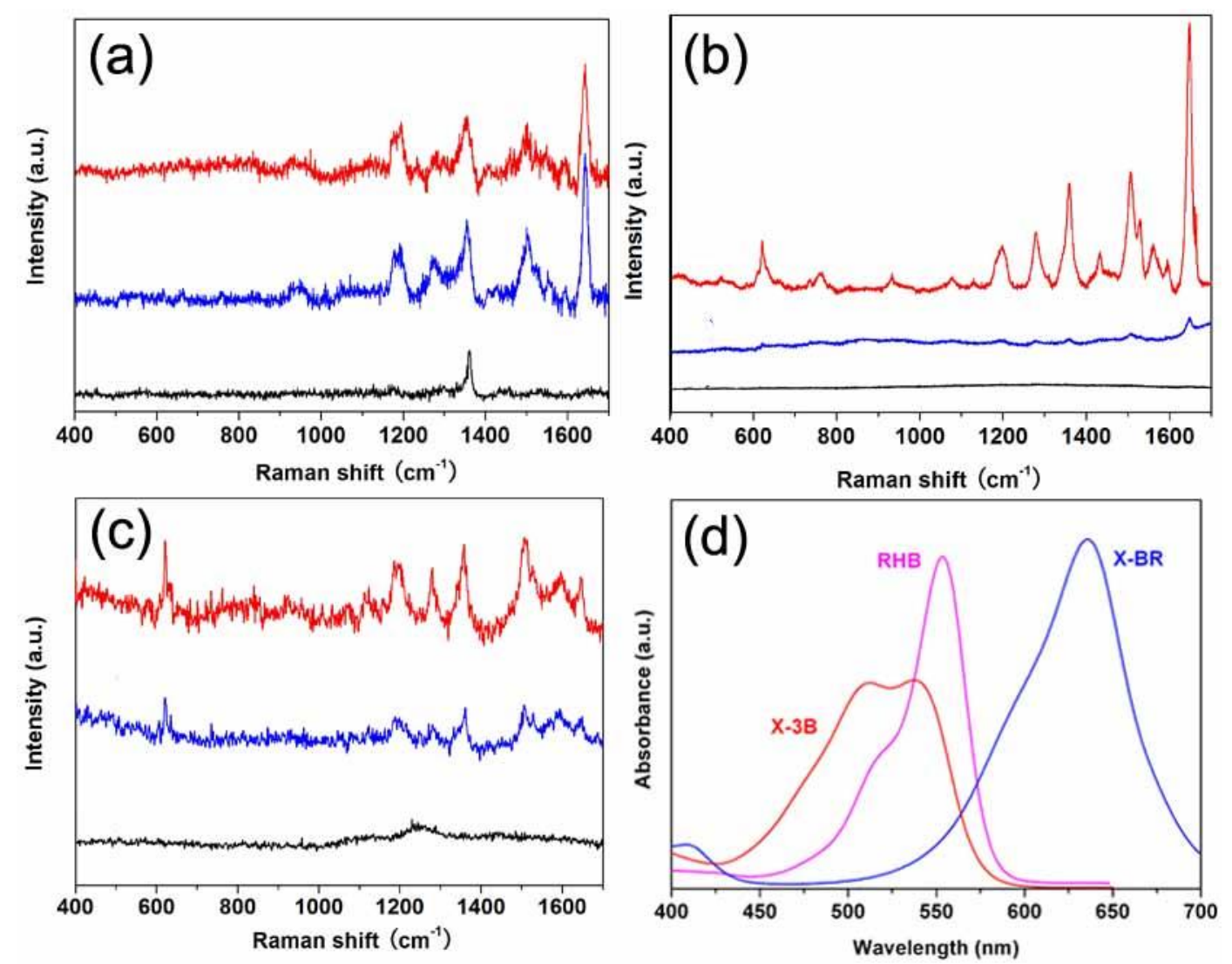

Fig. 9 Raman spectra of RHB on GC-TNFAs under different laser wavelengths (a) 473 nm, (b) $532 \mathrm{~nm}$ and (c) $785 \mathrm{~nm}$. Red line: GC-TNFAs, blue line: gold-coated aspolished pristine tungsten, black line: as-polished (no gold coating) tungsten substrates. (d) UV-vis spectra of 10 ppm RHB (pink), X-3B (red) and X-BR (blue) solution.

It is known that the surface plasmon resonance of gold plays a major role in the enhancement of the sensitivity of Raman scattering when it undergoes resonance with special wavelength of Raman reporters under special excitation laser source [38-40]. The SERS effect of gold is closely related to the material surface structure (e.g., geometry, morphology, etc.) [41]. As previous mentioned, the dimension of our GCTNFA is about $50 \mathrm{~nm}$. According to previous reports, for gold nanoparticles with diameters in the range of $10-100 \mathrm{~nm}$, the surface plasmon absorption band maxima was found in the region of $518 \mathrm{~nm}$ to $556 \mathrm{~nm}$, and peak center positions was particle size dependent [41]. The $532 \mathrm{~nm}$ excitation laser used in the present study, falls in the center of the reported wavelength region, indicating the plasmon resonance experienced by our GC-TNFAs, or more specifically, the gold films deposited on the tungsten 
nanofuzzes. The defined localized surface plasmon resonance of gold film on the TNFAs generated "hot spots" around the nanofuzz tips, resulting in highly sensitive SERS signals under the $532 \mathrm{~nm}$ laser.

According to the UV-vis spectrum in Fig 9(d), the absorption peak of RHB is 553 $\mathrm{nm}$, which is very close to that of the laser excitation wavelength $532 \mathrm{~nm}$. Since resonance enhancement does not begin at a sharply defined wavelength but can be observed when the exciting laser is within a few 100 wavenumbers below the electronic transition of a molecule [42]. A pre-resonance Raman effect can be expected occurred in detection of RHB samples, which further enhance the observed Raman spectra. Furthermore, the absorption peaks of X-3B and X-BR are observed at 538 and 636, respectively, which were not far from the laser excitation wavelength $532 \mathrm{~nm}$. Preresonance Raman effect can thus occur on these dye molecules. It is worth mentioning that, the TNFA produced in this work is not an ordered periodic structure and as a result, the sizes of surface Au nanostructures and gap in between are not homogeneous. However, given that the spot-size of the excitation beam $(\sim 2 \mu \mathrm{m})$ is much larger than the size of the nanofuzz tips $(\sim 50 \mathrm{~nm})$, the spatial averaging of hotspots on a macroscopic scale can make sure the efficiency of our TNFAs substrate in providing uniform SERS sensitivity over the large-surface area.

\section{Conclusions}

A highly sensitive SERS substrate with a novel gold-coated tungsten nanofuzz array structure has been developed and characterized in this study. The substrate exhibited strong SERS activity in detecting model RHB dye, with detection limit down to $10 \mathrm{ppb}$. Comparative studies indicated that the SERS activity of the SERS substrate is surface morphology dependent, which can be closely associated to the roughness and the specific surface area of the substrate. In addition, the SERS activity is found to be dependent on the laser excitation wavelength, with $532 \mathrm{~nm}$ laser showing the strongest efficiency in dye detection. The GC-TNFAS substrate has also been successfully 
deployed for the detection of other dye molecules commonly used in the industry. Finally, the SERS substrate could be reutilized after a simple plasma cleaning process. The design and development of our novel SERS substrate provides a simple and costeffective solution for the detection of environmental pollutants.

\section{Acknowledgment}

This work was supported financially by Natural Science Foundation of Liaoning Province (201602193), the NSFC (11204025, 11505025, 11475042), the National Magnetic Confinement Fusion Program (2015GB112001), the open project (Key Laboratory of Functional Materials Physics and Chemistry( Jilin Normal Univerisity) Ministry of Education,China (20162016003)) and the Fundamental Research Funds for the Central Universities DC201502080404.

\section{References}

[1] I. Holme, Sir William Henry Perkin: a review of his life, work and legacy. Color Technol, 122 (2006) 235-251.

[2] J. Liu, Y. Liu, N. Liu, Y. Han, X. Zhang, H. Huang, Y. Lifshitz, S. T. Lee, J. Zhong, Z. Kang, Metal-free efficient photocatalyst for stable visible water splitting via a two-electron pathway. Science. 347(2015) 970-974.

[3] X. Li, G. Chen, L.Yang, Z Jin, J. Liu, Multifunctional Au-coated $\mathrm{TiO}_{2}$ nanotube arrays as recyclable SERS substrates for multifold organic pollutants detection. Adv. Funct. Mater. 20 (2010) 2815-2824.

[4] Y.W. Liu, F. Luo, S.M. Liu, S.X. Liu, X.Y. Lai, X.H. Li,Y. Lu, Y.G. Li, C.W. Hu, Z. Shi and Z. P. Zheng, Aminated graphene oxide impregnated with photocatalytic polyoxometalate for efficient adsorption of dye pollutants and its facile and complete photoregeneration. Small. 13(2017) 1603174. 
[5] P.W. Li, F.L.Yuan, C. Z. Huang, Q. Zhang, Visual detection of sudan dyes based on the plasmon resonance light scattering signals of silver nanoparticles. Anal. Chem. 78(2006) 5570-5577.

[6] A.Sayari, S. Hamoudi, Y.Yang, Applications of pore-expanded mesoporous Silica1. Removal of heavy metal cations and organic pollutants from wastewater. Chem. Mater., 17 (2005) 212-216.

[7] C. Lofrumento, M. Ricci, E. Platania,M. Becucci, E.Castellucci. SERS detection of red organic dyes in Ag-agar gel. J. Raman Spectrosc . 44(2013) 47-54.

[8] M. Leona, Microanalysis of organic pigments and glazes in polychrome works of art by surface-enhanced resonance Raman scattering. Proc. Natl. Acad. Sci. USA 106(2009)14757-14762.

[9] Q.T. Zhou, Review of microfluidic approaches for surface-enhanced Raman scattering Review article. Sensor. Actuat. B-Chem. 227(2016) 504-514.

[10] M. Fleischmann,P. Hendra, A. McQuillan, Raman-spectra of pyridine adsorbed at a silver interface. Chem. Phys. Lett. 26(1974) 163-166.

[11] S.M. Nie, S.R. Emory, Probing Single Molecules and Single Nanoparticles by Surface-Enhanced Raman Scattering. Science 275(1997)1102-1106.

[12] H.W. Liu, I. Zhang, X. Lang,Y. Yamaguchi, H. Iwasaki,;Y. Inouye, Q. Xue, M. Chen, Single molecule detection from a large-scale SERS-active $\mathrm{Au}_{79} \mathrm{Ag}_{21}$ substrate. Sci. Rep. 1(2011)112.

[13] S. Shanmukh, L. Jones, J. Driskell, Y. P. Zhao,R. Dluhy, R. A. Tripp, Rapid and Sensitive Detection of Respiratory Virus Molecular Signatures Using a Silver Nanorod Array SERS Substrate. Nano Lett., 6(2006) 2630-2636.

[14] J. W. Jeong, M. M. P. Arnob,K. M. Baek, S. Y. Lee, W. C. Shih, Y. S. Jung, 3D cross-point plasmonic nanoarchitectures containing dense and regular hot spots for surface-enhanced Raman spectroscopy analysis. Adv. Mater. 28(2016) 8695-8704. 
[15] Y. Shin,J. H. Song,D.C. Kim, Facile Preparation of ultrasmall void metallic nanogap from self-assembled gold-Silica core-shell nanoparticles monolayer via kinetic control. Adv. Mater. 27(2015) 4344-4350.

[16] P. G. Etchegoin,;E.C. L. Ru, A perspective on single molecule SERS: current status and future challenges. Phys. Chem. Chem. Phys. 10 (2008) 6079-6089.

[17] D. P. Fromn, A. Sundaramrthy,P. J. Schuck, G. S. Kino,W. E. Moerner, Exploring the chemical enhancement for surface-enhanced Raman scattering with Au bowtie nanoantennas. J. Chem. Phys. 124 (2006) 061101.

[18] F.J.Garcia-Vidal, J.B. Pendry, Collective theory for surfaceenhanced Raman scattering. Phys. Rev. Lett. 77(1996)1163-1166.

[19] E.C. LeRu, P.G. Etchegoin, Principles of Surface-Enhanced Raman Spectroscopy. Elsevier 1(2009) 50.

[20] H. Ko, S. Singamaneni, V. V. Tsukruk, Nanostructured surfaces and assemblies as SERS media. Small. 4(2008)1576-1599.

[21] P. Zheng, S. K. Cushing, S. Suri, N.Q Wu, Tailoring plasmonic properties of gold nanohole arrays for surface-enhanced Raman scattering. Phys. Chem. Chem. Phys., 17 (2015) 21211.

[22] G. Luca, V. G. R. Jose, D. Concepcion and S.C. Santiago, Ultrathin silver-coated gold nanoparticles as suitable substrate for surface-enhanced Raman scattering. J. Raman Spectrosc. 41 (2010) 508-515.

[23] K. Vijayarangamuthu, S. Rath, One-step synthesis of Au-coated porous silicon as a surface enhanced Raman scattering substrate for biomolecule detection. Mater. Lett. 204 (2017) 115-119.

[24] J. G.Fan, Y. P. Zhao, Gold-coated nanorod arrays as highly sensitive substrates for surface-enhanced Raman spectroscopy. Langmuir, 24 (2008) 14172-14175.

[25] M. C. Wu, M.P. Lin, S. W. Chen, P.H. Lee, J.H. Li, W. F. Su. Surface-enhanced 
Raman scattering substrate based on a Ag coated monolayer array of $\mathrm{SiO}_{2}$ spheres for organic dye detection. RSC Adv., 4 (2014) 10043-10050.

[26] Q. Yang, Y. W. You, L. Liu, H.Y. Fan, W. Y. Ni, D. P. Liu, C. S. Liu, Nanostructured fuzz growth on tungsten under low-energy and high-flux He irradiation. Sci. Rep. $5(2015) 10959$.

[27] L. Liu, D.P. Liu,Y. Hong, H.Y. Fan, W.Y. Ni, Q. Yang, Z.H. Bi, G. Benstetter, S. Li, High-flux $\mathrm{He}+$ irradiation effects on surface damages of tungsten under ITER relevant conditions. J. Nucl. Mater. 471(2016)1-7.

[28] X.Y. Tan, L.M. Luo, H.Y. Chen, X.Y. Zhu, X. Zan, G.G. Luo, J.L. Chen, P. Li, J.G. Cheng, D.P. Liu, Y.C. Wu, Mechanical properties and microstructural change of $\mathrm{W}-\mathrm{Y}_{2} \mathrm{O}_{3}$ alloy under helium irradiation. Sci. Rep. 5 (2015)12755.

[29] X. Huang, S.Z Li, S.X. Wu, Y.Z. Huang, F. Boey, C. L. Gan, H. Zhang, Graphene Oxide-Templated Synthesis of Ultrathin or Tadpole-Shaped Au Nanowires with Alternating hep and fcc Domains. Adv. Mater. 24 (2012) 979-983.

[30] K. Kasinathan,J.Kennedy, M. Elayaperumal, M. Henini, M. Malik, Photodegradation of organic pollutants RhB dye using UV simulated sunlight on ceria based $\mathrm{TiO}_{2}$ nanomaterials for antibacterial applications. Sci. Rep. 6(2016) 38064 .

[31] X.M Zhou,.J.Y. Lan, G. Liu, K. Deng, Y.L. Yang, G.J. Nie, J.G. Yu, L.J. Zhi, FacetMediated Photodegradation of Organic Dye over Hematite Architectures by Visible Light. Angew. Chem. 124 (2012) 182-186.

[32] H.P. Yang, H.L.Hu, Z.H. Ni, C. K. Poh, C.X. Cong,J.Y. Lin, T. Yu, Comparison of surface-enhanced Raman scattering on graphene oxide, reduced graphene oxide and graphene surfaces. Carbon, 62(2013) 422-429.

[33] Y. Zhao, W.C. Zeng, Z.C. Tao, P.H. Xiong, Y. Qu, Y.W. Zhu, Highly sensitive surface-enhanced Raman scattering based on multi-dimensional plasmonic coupling in $\mathrm{Au}$-graphene-Ag hybrids. Chem. Commun., 51(2015) 866-869. 
[34] Y. Song, W.C. Wang, D.P. Liu, Q. Zhang, W.Y. Ni, J.H. Niu. The Pebrine disease of Chinese silkworm controlled by using atmospheric cold plasma jet. IEEE. T. Plasma. Sci. 41( 2013) 2370-2376.

[35] Z.X. Xu, J. Shen, C. Cheng, S.H. Hu, Y. Lan, P. K. Chu, J. Phys. D. In vitro antimicrobial effects and mechanism of atmospheric-pressure $\mathrm{He} / \mathrm{O}_{2}$ plasma jet on Staphylococcus aureus bio film. Appl. Phys 50(2017) 105201.

[36] D. X. Liu, M.Z. Rong, X.H.Wang, F. Iza, M.G. Kong, P. Bruggeman, Main species and physicochemical processes in cold atmospheric-pressure $\mathrm{He}+\mathrm{O}_{2}$ Plasmas. Plasma. Process. Polym. 7 (2010) 846-865.

[37] F.X. Yang,Q. Zhao,C.H. Xu,Y. Zou, H.L.Dong, Y.G. Zheng, W.P. Hu, Organic Electronics: Unveiling the switching riddle of silver tetracyanoquinodimethane towards novel planar single-crystalline electrochemical metallization memories. Adv. Mater. 28(2016) 7031 .

[38] V. Amendola, S. Scaramuzza, S. Agnoli, S. Polizzi, M. Meneghetti, Strong dependence of surface plasmon resonance and surface enhanced Raman scattering on the composition of Au-Fe nanoalloys. Nanoscale , 6 (2014) 1423-1433.

[39] A. Samanta, K. K. Maiti, K. S. Soh, X. J. Liao, M.Vendrell,U. S. Dinish, S. W. Yun, R. Bhuvaneswari,H. Kim, S. Rautela, J. H. Chung, M. Olivo, Y. T. Chang, Ultrasensitive near-infrared Raman reporters for SERS-based in vivo cancer detection. Angew. Chem., Int. Ed., 50 (2011) 6089-6092.

[40] A. Samanta, S. Jana, R. K. Das, Y.T. Chang. Wavelength and shape dependent SERS study to develop ultrasensitive nanotags for imaging of cancer cells. RSC Adv., $4(2014)$ 12415-12421.

[41] N. G. Bastus, J. Comenge, V. Puntes, Kinetically Controlled Seeded Growth Synthesis of Citrate-Stabilized Gold Nanoparticles of up to $200 \mathrm{~nm}$ : Size Focusing versus Ostwald Ripening. Langmuir, 27(2011) 11098-11105.

[42] A.Y. Hirakawa, M. Tsuboi, Molecular geometry in an excited electronic state 
and a preresonance Raman effect. Science, 188 (1975) 359-361. 